\title{
High control in high power fiber lasers and amplifiers
}

Y. Jeong, J. N. Maran, S. Yoo, A. J. Boyland, J. K. Sahu, and J. Nilsson

Optoelectronics Research Centre, University of Southampton, Highfield, Bldg 46, Southampton SO17 1BJ, UK, Tel: +44 238059 3141, Fax: +44 238059 3142, Email: yoj@orc.soton.ac.uk

Abstract We discuss fundamental aspects and future prospects of high power fiber lasers and amplifiers with particular attention to high control of cladding-pumped, rare-earth-doped fiber sources in a multitude of regimes.

\section{Summary}

The past few years have seen dramatic advances in fiber laser and amplifier technologies. Fiber lasers of output powers beyond a kilowatt or multi-kilowatt with high beam quality have already been demonstrated and started to be deployed in industries [1]-[3]. This rapid progress has been stimulated by the exceptional power-handling ability of rare-earth-doped fibers and the availability of powerful diode lasers for use as pumps. Indeed, this fiber circuitry combined with pump-diode technology provides a unique highgain environment for robust designs, which is also allsolid state, compact, stable, reliable, and reproducible. Furthermore, it can also provide a variety of powerful solutions to even more refined sources, such as single-frequency, tunablefrequency, plane-polarized, or pulsed sources with various repetition rates and durations [4], [5].

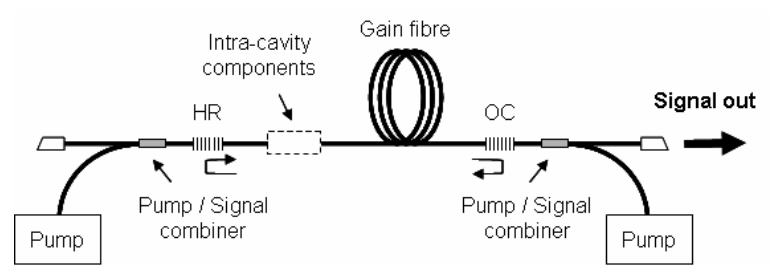

(a)

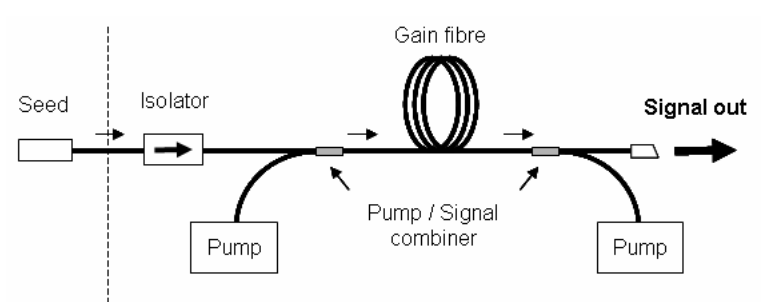

(b)

Fig. 1. (a) Laser (Oscillator) configuration, (b) MOPA configuration
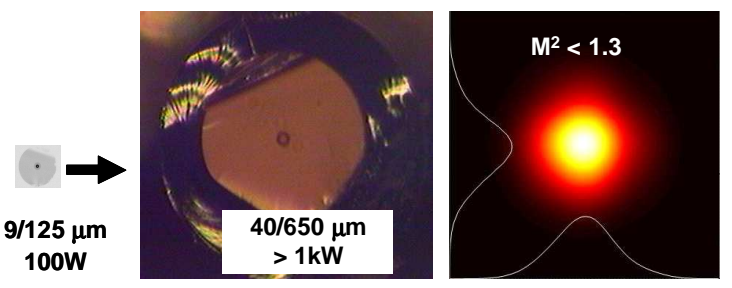

Fig. 2. Images of doped fibers used for high power and typical output beam profile from a large-core fiber
Figure 1 shows typical schematic diagrams for fiber laser and fiber master-oscillator power amplifier (MOPA) configurations. As the output power of such fiber sources grows, even more attention needs to be paid to potential limitation factors, such as material damage threshold, nonlinear scatterings, thermal distortion, photo-darkening, etc. The management of beam quality is also a big concern because highpower fibers normally use multi-mode large core designs, as depicted in Fig. 2, unlike most fibers used in optical telecoms. Consequently, technological advances in fiber design, pump coupling scheme, mode control, heat dissipation, output coupling, suppression of nonlinear scatterings (e.g. SBS, SRS, SPM, and XPM) and add-on options for the spectral or temporal control are the key issues for such highpower sources. Figure 3 shows the output power characteristics of our recent result on an erbium:ytterbium co-doped fiber in a laser configuration, for example. While laser (oscillator) configurations themselves are very effective in extracting high output power via relatively simple and compact arrangement, MOPA configurations can provide easy and excellent control of precise output characteristics because they allow the seed sources that are in low power to be designed for controllability and precision, while amplification to high power can be designed into the power amplifiers that can also be constructed in multiple stages. Thus, these are often employed for highly refined high power output with options for single-frequency, frequency-tuning, pulsed operation, etc.

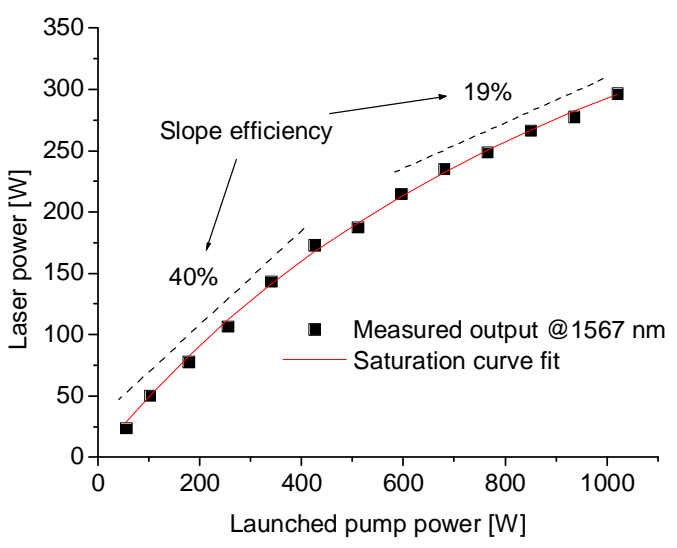

Fig. 3. Erbium:ytterbium co-doped large-core fiber 
laser with 297 W (54.7 dBm) continuous-wave output power. The maximum output power was limited by the available pump power; however, the slope efficiency with respect to the launched pump power changed from $40 \%$ to $19 \%$ at higher output power due to the onset of $\mathrm{Yb}$ co-lasing at $1067 \mathrm{~nm}$.

Here we review the recent progress in high power lasers and amplifiers based on rare-earth-doped fibers and discuss their fundamental and high control aspects. We also present our up-to-date experimental results with particular attention to a multitude of cladding-pumped, ytterbium-doped fibers for $1.1 \mu \mathrm{m}$ emissions and erbium-ytterbium co-doped fibers for $1.5 \mu \mathrm{m}$ emissions. These include a multi-kilowatt continuous-wave fiber laser, single-frequency MOPA sources, pulsed fiber MOPA sources in various repetition and duration regimes, and their further applications to nonlinear wavelength conversions.

\section{References}

1 Y. Jeong et al., Opt. Express, vol. 12, no. 25, pp. 6088-6092, 2004.

2 A. Liem et al., CLEO 2004, postdeadline paper CPDD2.

3 V. Gapontsev et al., CLEO Europe 2005, paper CJ1-1-THU.

4 Y. Jeong et al., Opt. Lett., vol. 30, no. 22, pp. 29972999, 2005.

5 P. Dupriez et al., IEEE Photon. Technol. Lett., vol. 18, no. 9, pp. 1013-1015, 2006. 